\title{
Accurate quantitative measurements of brachial artery cross-sectional vascular area and vascular volume elastic modulus using automated oscillometric measurements: comparison with brachial artery ultrasound
}

\author{
Yuuki Tomiyama ${ }^{1}$, Keiichiro Yoshinaga ${ }^{2}$, Satoshi Fujii ${ }^{3}$, Noriki Ochi ${ }^{4}$, Mamiko Inoue ${ }^{5}$, Mutumi Nishida ${ }^{1}$, \\ Kumi Aziki ${ }^{2}$, Tatsunori Horie ${ }^{5}$, Chietsugu $\mathrm{Katoh}^{4}$ and Nagara Tamaki ${ }^{1}$
}

Increasing vascular diameter and attenuated vascular elasticity may be reliable markers for atherosclerotic risk assessment. However, previous measurements have been complex, operator-dependent or invasive. Recently, we developed a new automated oscillometric method to measure a brachial artery's estimated area $(\mathrm{eA})$ and volume elastic modulus $\left(V_{\mathrm{E}}\right)$. The aim of this study was to investigate the reliability of new automated oscillometric measurement of eA and $V_{\mathrm{E}}$. Rest eA and $V_{\mathrm{E}}$ were measured using the recently developed automated detector with the oscillometric method. eA was estimated using pressure/volume curves and $V_{E}$ was defined as follows ( $V_{E}=\Delta$ pressure/ $(100 \times \Delta$ area/area) $\mathrm{mm} \mathrm{Hg} / \%$ ). Sixteen volunteers (age $35.2 \pm 13.1$ years) underwent the oscillometric measurements and brachial ultrasound at rest and under nitroglycerin (NTG) administration. Oscillometric measurement was performed twice on different days. The rest eA correlated with ultrasound-measured brachial artery area $(r=0.77, P<0.001)$. Rest $\mathrm{eA}$ and $\mathrm{V}_{\mathrm{E}}$ measurement showed good reproducibility (eA: intraclass correlation coefficient $\left.(I C C)=0.88, V_{E}: I C C=0.78\right)$. Under NTG stress, eA was significantly increased $\left(12.3 \pm 3.0\right.$ vs. $\left.17.1 \pm 4.6 \mathrm{~mm}^{2}, P<0.001\right)$, and this was similar to the case with ultrasound evaluation $(4.46 \pm 0.72$ vs. $4.73 \pm 0.75 \mathrm{~mm}, P<0.001)$. $V_{E}$ was also decreased $(0.81 \pm 0.16$ vs. $0.65 \pm 0.11 \mathrm{~mm} \mathrm{Hg} / \%, P<0.001)$ after NTG. Cross-sectional vascular area calculated using this automated oscillometric measurement correlated with ultrasound measurement and showed good reproducibility. Therefore, this is a reliable approach and this modality may have practical application to automatically assess muscular artery diameter and elasticity in clinical or epidemiological settings.

Hypertension Research (2015) 38, 478-484; doi:10.1038/hr.2015.6; published online 19 February 2015

Keywords: brachial artery; cross-sectional area; elasticity; oscillometric; ultrasound

\section{INTRODUCTION}

Arterial diameter enlargement is thought to be an early indicator in the progression of atherosclerosis. ${ }^{1,2}$ Increasing brachial artery diameter is related to conventional cardiovascular risk factors. ${ }^{3}$ Brachial artery ultrasound has been used to obtain this measurement and is considered to be the standard measurement. However, data acquisition and data analysis require experienced operators, and therefore an alternative simple automated measurement approach should be developed.

Functional deterioration appears before the development of anatomical vascular changes. ${ }^{4-6}$ Arterial elastic mechanics are linked to arterial stiffness. ${ }^{7-9}$ Determination of arterial diameter at very low or zero transmural pressure makes calculation of vessel strain and volume elastic modulus $\left(V_{\mathrm{E}}\right)$ possible. ${ }^{10}$ Some studies have estimated the $V_{\mathrm{E}}$ in different approaches. Bank et al. ${ }^{10}$ estimated this marker using a water-filled blood pressure (BP) cuff with an external ultrasound. Kinlay et al. estimated this marker using intravascular ultrasound and a catheter. ${ }^{11}$ Both approaches measured vascular area changes during various pressure changes. Kinlay et al. ${ }^{12}$ obtained intraarterial pressure from the side arm of the arterial sheath, and Bank et al. ${ }^{10}$ obtained arterial pressure information using applanation tonometry. These earlier approaches were either complex or invasive, and hence a simple noninvasive $V_{\mathrm{E}}$ measurement that could be widely used in clinical settings was sought. Recently, we developed a new automated

${ }^{1}$ Department of Nuclear Medicine, Hokkaido University Graduate School of Medicine, Hokkaido, Japan; ${ }^{2}$ Department of Molecular Imaging, Hokkaido University Graduate School of Medicine, Hokkaido, Japan; ${ }^{3}$ Department of Laboratory Medicine, Asahikawa Medical University, Hokkaido, Japan; ${ }^{4}$ Faculty of Health Sciences, Hokkaido University Graduate School of Medicine, Hokkaido, Japan and ${ }^{5}$ Division of Clinical Laboratory and Transfusion Medicine, Hokkaido University Hospital, Hokkaido, Japan

Correspondence: Dr K Yoshinaga, Department of Molecular Imaging Research, National Institute of Radiological Science, 4-9-1 Anage, Inage-Ku, Chiba 263-8555, Japan. E-mail: kyoshi@nirs.go.jp

Received 30 June 2014; revised 11 November 2014; accepted 31 December 2014; published online 19 February 2015 
oscillometric method to measure a brachial artery's estimated area (eA) and $V_{\mathrm{E}}$. The main control unit created various fixed precise volumes of air that are capable of changing the tube size, giving rise to the so-called tube law. ${ }^{13}$ Changing tube size may be associated with vascular cross-sectional area. Differences between intramural BP and cuff pressure $(\mathrm{CP})$ can be obtained using oscillometric measurements. This is the fundamental concept of this automated system. Using the pressure/volume curves, this oscillometric method quantitatively estimates eA and $V_{\mathrm{E}}$. Preliminary data by Otsuka et al. ${ }^{14}$ showed a close relationship between eA and cardiovascular risk factors during health checkups. However, this approach has not been validated using other standard measurements, and its reproducibility has not been evaluated.

The aim of this study was to investigate the reliability of the new automated and quantitative oscillometric measurement of eA and $V_{\mathrm{E}}$. Therefore, we compared eA with ultrasound measurements as a standard and evaluated the reproducibility of these measurements.

\section{METHODS}

\section{Study subjects}

Sixteen volunteers participated in the study. Volunteers were recruited from our Hokkaido University Hospital staff members, Postgraduate School of Medicine staff members and Department of Health Sciences, Hokkaido University staff members. All participants were men and two participants were cigarette smokers. All participants had a normal resting electrocardiogram and did not have a history of cardiovascular disease. They were not taking any cardiac medications. Fourteen healthy control subjects had a low pretest likelihood of coronary artery disease $(<5 \%)$ based on risk factors. ${ }^{15}$

Among 16 healthy individuals, 8 subjects $\leqslant 35$ years of age were classified as the younger group. The remaining 8 who were $>35$ years of age were categorized as the older group. ${ }^{16}$

The study was approved by the Hokkaido University Graduate School of Medicine Human Research Ethics Board. Written informed consent was obtained from all subjects.

\section{Study protocol}

We evaluated vascular function using brachial artery ultrasound and automated oscillometric methods at rest and after sublingual nitroglycerin (NTG) administration. In the ultrasound study, we obtained data at rest, after flowmediated dilatation and after NTG administration. However, we used only rest and NTG stress data in this study because we did not perform reactive hyperemia for oscillometric measurements.

We also performed oscillometric measurements twice, on different days, to evaluate the reproducibility of this automated technique. These measurements were performed in randomized order. All examinations were performed within a 3-week period.

We obtained blood samples on the day of the first oscillometric measurements.

\section{Blood sampling and biochemical measurements}

We performed venous blood sampling after the oscillometric measurement under overnight fasting conditions. ${ }^{17,18}$ Fasting blood sugar was measured using the glucose oxidase method. Serum cholesterol and triglycerides were measured using standard enzymatic methods. High-density lipoprotein cholesterol concentration was measured from the serum supernatant after precipitation of very-low-density lipoprotein and low-density lipoprotein subfraction. Lowdensity lipoprotein cholesterol was calculated by the Friedewald formula. ${ }^{19}$

\section{Brachial artery ultrasound measurements with flow-mediated vasodilatation: subject preparation}

Participants were instructed to abstain from caffeine-containing products (coffee, tea, chocolate and cola) for at least $24 \mathrm{~h}$ and vitamin C-containing beverages for at least $6 \mathrm{~h} .{ }^{5}$ They fasted for $\geqslant 8 \mathrm{~h}$ (overnight fasting) before the ultrasound measurements. ${ }^{11,20}$ Two smokers were instructed to abstain from smoking for at least $12 \mathrm{~h}$ in order to minimize acute smoking effects. The ultrasound study was conducted in the morning $(0800 \mathrm{~h})$ after overnight fasting (Figure 1a). Subjects rested in a supine position for $10 \mathrm{~min}$ before the study in a temperature-controlled room $\left(21\right.$ to $\left.23^{\circ} \mathrm{C}\right)$. An automated sphygmomanometer cuff was positioned on the left arm for measurement of BP..$^{11}$

\section{Image acquisition}

High-resolution Doppler ultrasonography equipment (Aplio XG, Toshiba, Tokyo, Japan) with an $18-\mathrm{MHz}$ transducer was used to evaluate the right brachial artery. The onset of the $\mathrm{R}$ wave was used to identify end-diastolic vascular diameter, and the peak of the $\mathrm{T}$ wave was used to identify end-systolic diameter. $^{21}$

The brachial artery was longitudinally scanned $\sim 5 \mathrm{~cm}$ above the antecubital fossa. We also evaluated brachial artery diameter $8.5 \mathrm{~cm}$ above the antecubital fossa before the standard rest measurement, which was $5 \mathrm{~cm}$ above the antecubital fossa, in order to compare oscillometric measurements. After an appropriate probe position was determined, the skin was marked and the arm was retained in the same position throughout the study. The echo transducer was supported by a stereotactic probe-holding device.

After $10 \mathrm{~min}$ of rest in a supine position, brachial artery diameter was measured at rest as a baseline study.

\section{Endothelium-independent vasodilatation with NTG}

At $15 \mathrm{~min}$ after the flow-mediated dilatation study, a new baseline image was obtained. After the second baseline data acquisition, subjects received $0.3 \mathrm{mg}$ of sublingual NTG (TOA EIYO, Fukushima, Japan). Just after sublingual NTG administration, brachial artery image acquisition was started and continued for 5 min. $^{11}$

\section{Image analysis}

Brachial artery diameter was measured from the anterior to the posterior medial-adventitial interface at the end-diastole. We evaluated the vascular diameter for four cardiac cycles and calculated the mean value. The means of the two measurements by independent observers were calculated. The interoperator reproducibility of ultrasound measurement in our facility had an intraclass correlation coefficient (ICC) of 0.92 . The percentage increase of diameter by NTG (\%NTG) was calculated as follows: ${ }^{22}$

$\% \mathrm{NTG}=$ brachial artery diameter at NTG stress/average of baseline diameter $\times 100$.
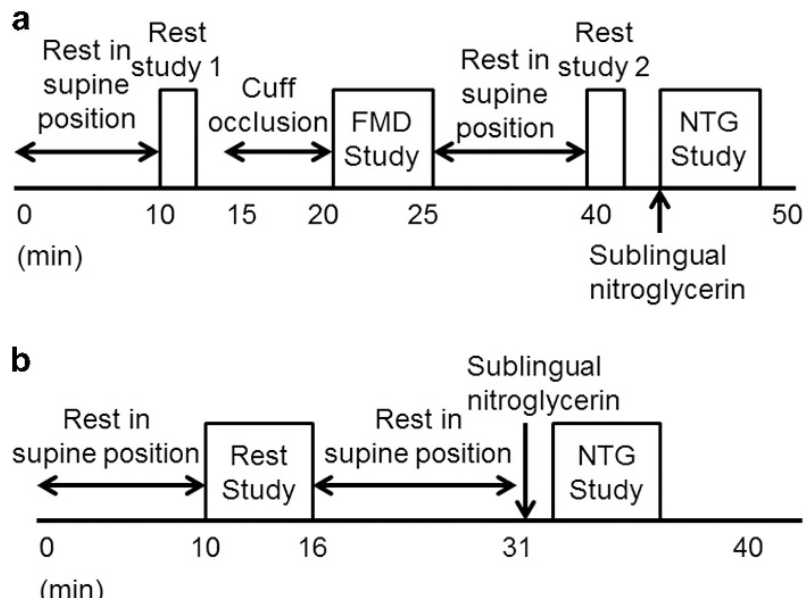

Figure 1 Study design. (a) Data acquisition protocol of ultrasound measurements. (b) Data acquisition protocol of oscillometric measurements. FMD, flow-mediated dilatation; NTG, nitroglycerin. 


\section{Automated vascular volume/pressure detector measurements:} oscillometric measurement protocol

Subjects followed the same instructions they had received for ultrasound measurements before the studies. Participants underwent oscillometric measurements after overnight fasting. We performed rest brachial arterial crosssectional area and functional measurements using a newly developed device, the Health Chronos TM-2771 prototype (A\&D Company, Tokyo, Japan) (Figure 2a). ${ }^{14}$ Data acquisition took 6 min (Figure 1) and included measurements of $\mathrm{BP}$, eA and $V_{\mathrm{E}}$ (that is, vascular stiffness). After the rest data acquisitions, participants rested for $15 \mathrm{~min}$. Then, subjects received sublingual NTG $(0.3 \mathrm{mg})$. At $2 \mathrm{~min}$ after sublingual NTG administration, the second measurements were performed (Figure 1b).

a

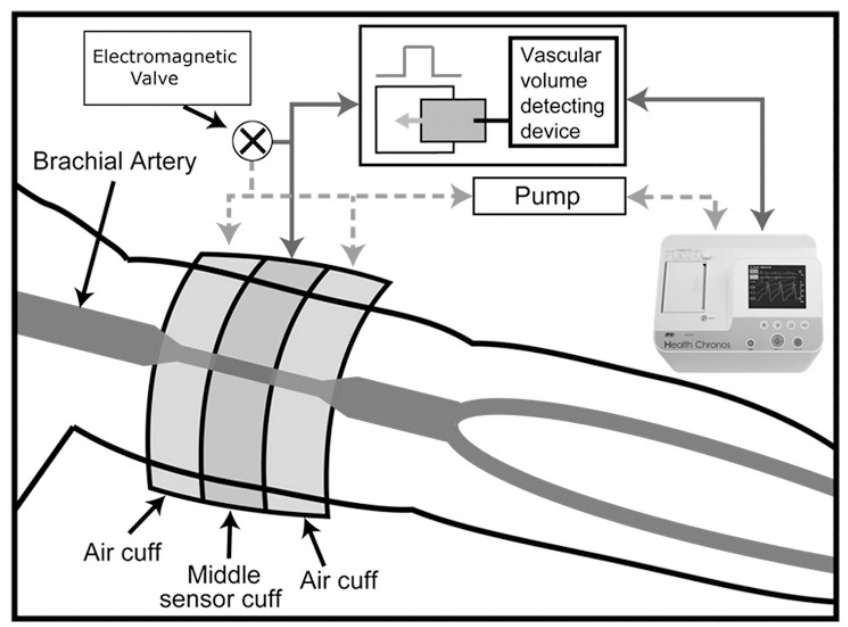

b

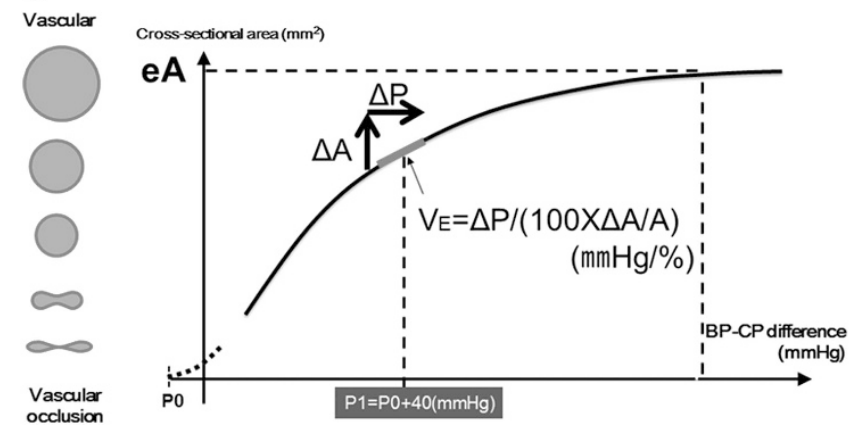

Figure 2 (a) Oscillometric measurement set-up. A triple lumen cuff was put on the upper arm. The triple lumen cuff included central pressure sensor cuff and two outside cuffs. The central part of the triple lumen cuff detected the exact vascular volume change. A main control unit generated a precise amount of air into all three cuffs. After sending the precise amount of air, two tubes to the outside cuffs were occluded. These amounts of air changed the vascular volume and added a stable pressure to the vessel. After sending the air, the main control unit changed a valve connection to the central cuff and the central cuff changed its role. The central cuff then sensed the vascular volume change and controlled the cuff pressure. The central pressure sensor cuff detected BP-CP differences during heartbeats. Using this information, the main control unit made pressure/volume curves. (b) Calculation of estimated cross-sectional area (eA) and volume elastic modulus $\left(V_{E}\right)$ using pressure/volume curves. When cuff pressure became zero, the calculated cross-sectional vascular area represented baseline vascular area. BP, blood pressure; $\mathrm{CP}$, cuff pressure. A full color version of this figure is available at the Hypertension Research journal online.

\section{Data analysis}

We measured brachial artery absolute volume and $V_{\mathrm{E}}$ using a newly developed device, the Health Chronos TM-2771 prototype. Details of this device are addressed elsewhere. ${ }^{14}$ In brief, this device consists of a main control unit and two outside cuffs for oscillometric measurement and plethysmography at the right and left brachial arteries (Figure 2a). The exteriors of the three occlusive lumen cuffs are composed of a hard material to avoid expansion with increases in arterial volume. Therefore, the $\mathrm{CP}$ changes accurately reflected volumetric changes of the brachial artery. To measure a brachial artery's absolute volume, the device initially sought the lowest $\mathrm{CP}$ that indicated complete occlusion of the brachial artery (volume $=0$ ) at end-diastole. During the measurement, the main control unit put air into the central cuff for a calibration. This calibration made it possible to determine the absolute volumetric changes of the brachial artery from the plethysmogram under the condition of outside cuff inflation. The CP was gradually decreased and the calibrated plethysmogram was recorded several times at regular $\mathrm{CP}$ intervals. Thus, pressure and area curves were obtained.

To estimate the cross-sectional area of the brachial artery, the brachial arterial volume was divided by the length of the central cuff, which was a pressure-sensor cuff.

The eA was defined as the estimated cross-sectional area at the point where the BP-CP difference was identical to the diastolic BP (Figure 2b). The highest $\mathrm{CP}$, indicating complete collapse of the brachial artery, is usually a little higher than BP. Therefore, the corresponding point (P0) on the pressure axis of the curve was set at less than zero.

$V_{\mathrm{E}}$ was defined as the change in the BP-CP difference (DP1) per $1 \%$ increase in cross-sectional area at $\mathrm{P} 1$, where $\mathrm{P} 1=\mathrm{P} 0+40 \mathrm{~mm} \mathrm{Hg}$. The original calculation of $V_{\mathrm{E}}$ was as follows: ${ }^{23}\left(V_{\mathrm{E}}=\Delta\right.$ Pressure/ $(100 \times \Delta$ volume/volume $)$ $\mathrm{mm} \mathrm{Hg} / \%)$. This system estimated the absolute value of cross-sectional vascular area instead of vascular volume. Thus, the original $V_{\mathrm{E}}$ equation was modified as follows: ${ }^{14}\left(V_{\mathrm{E}}=\Delta\right.$ pressure/ $(100 \times \Delta$ area/area $\left.) \mathrm{mm} \mathrm{Hg} / \%\right)$. The principal concept of $V_{\mathrm{E}}$ estimation was to evaluate the association between $\Delta \mathrm{A}$ and $\Delta \mathrm{P}$ while the blood vessel remained circular. The correct measurements should be performed with cuff pressure below a certain point so that the vessel remains circular and does not have buckling. Based on our basic preliminary experimental laboratory data analysis, in most cases vessels remained circular when pressure was $40 \mathrm{~mm} \mathrm{Hg}$ higher than $\mathrm{P} 0$.

Although we obtained the values of eA and $V_{\mathrm{E}}$ for both right and left brachial arteries, the values for eA and $V_{\mathrm{E}}$ for the right brachial artery were used in the subsequent statistical analysis as the ultrasound measurements we performed were for the right brachial artery.

The percentage increase of eA and $V_{\mathrm{E}}$ with NTG (\%NTG) was calculated as follows: $\% \mathrm{NTG}=\mathrm{eA}$ or $V_{\mathrm{E}}$ at NTG stress/rest eA or $V_{\mathrm{E}} \times 100$.

The measurement was similar to that obtained using ultrasound.

\section{Statistical analysis}

Continuous variables were presented as means and s.d. Categorical data were expressed as a percentage of total. The paired Student's $t$-test and unpaired Student's $t$-test were used for continuous variables. Pearson's correlation coefficient was analyzed to determine the simple correlation between variables. In the analysis, all statistical tests were two sided. A $P$-value of ${ }^{<} 0.05$ was considered indicative of a statistically significant difference. The reliability of oscillometric measurement was assessed using an ICC. The strength of the ICC was determined using the cutoffs of $0.5,0.3$ and 0.1 for high, moderate and low levels of agreement according to Cohen's effect size convention. ${ }^{23}$ Statistical calculations were carried out using SAS software version 9.2 (SAS Institute, Cary, NC, USA).

\section{RESULTS}

\section{Reliability of oscillometric measurements}

Subject characteristics. The baseline characteristics of the 16 subjects are shown in Table 1a. Among 16 individuals, 2 subjects were cigarette smokers. Lipid profiles, including total cholesterol and low-density cholesterol, fasting blood sugar and hemoglobin Alc, were within 
Table 1a Baseline characteristics

\begin{tabular}{lc}
\hline & Subjects $(\mathrm{n}=16)$ \\
\hline Normal/smoker & $14 / 2$ \\
Age (years) & $35.2 \pm 13.1$ \\
Height (cm) & $169.4 \pm 4.4$ \\
Weight (kg) & $68.0 \pm 9.2$ \\
BMI (kg m-2) & $23.7 \pm 3.2$ \\
Systolic BP (mm Hg) & $115.2 \pm 15.1$ \\
Diastolic BP (mm Hg) & $72.5 \pm 11.7$ \\
HR (b.p.m.) & $62.1 \pm 9.8$ \\
T-CHO (mg dl-1) & $199.3 \pm 39.9$ \\
LDL-CHO (mg dl & -1 ) \\
FBS (mg dl-1) & $119.1 \pm 62.9$ \\
HbAlc (\%) & $88.3 \pm 8.7$ \\
\end{tabular}

Abbreviations: BMI, body mass index; BP, blood pressure; FBS, fasting blood sugar; $\mathrm{HbA1c}$, hemoglobin Alc; HR, heart rate; LDL-CHO, low-density lipoprotein cholesterol; T-CHO, total cholesterol.

Data are presented as mean \pm s.d

Table 1b Baseline characteristics of younger and older groups

\begin{tabular}{|c|c|c|c|}
\hline & Younger $(\mathrm{n}=8)$ & Older $(\mathrm{n}=6)$ & $\mathrm{P}$-value \\
\hline Age (years) & $23.0 \pm 1.2$ & $44.3 \pm 5.8$ & $<0.001$ \\
\hline Height (cm) & $169.4 \pm 4.1$ & $169.3 \pm 5.8$ & 0.99 \\
\hline Weight (kg) & $66.6 \pm 9.6$ & $68.5 \pm 10.3$ & 0.72 \\
\hline $\mathrm{BMI}\left(\mathrm{kg} \mathrm{m}^{-2}\right)$ & $23.3 \pm 3.5$ & $23.9 \pm 5.8$ & 0.73 \\
\hline Systolic BP (mm Hg) & $118.1 \pm 17.0$ & $107.8 \pm 6.6$ & 0.18 \\
\hline Diastolic BP (mm Hg) & $72.3 \pm 10.6$ & $68.7 \pm 9.1$ & 0.52 \\
\hline HR (b.p.m.) & $66.2 \pm 10.6$ & $56.7 \pm 7.5$ & 0.09 \\
\hline $\mathrm{T}-\mathrm{CHO}\left(\mathrm{mg} \mathrm{dl}^{-1}\right)$ & $193.3 \pm 32.6$ & $204.5 \pm 50.2$ & 0.61 \\
\hline LDL-CHO (mg dl-1) & $109.6 \pm 16.2$ & $129.5 \pm 53.7$ & 0.33 \\
\hline FBS (mg dl-1) & $88.3 \pm 9.3$ & $87.5 \pm 7.0$ & 0.87 \\
\hline HbAlc (\%) & $4.9 \pm 0.2$ & $5.0 \pm 0.2$ & 0.23 \\
\hline
\end{tabular}

Abbreviations: $\mathrm{BMI}$, body mass index; $\mathrm{BP}$, blood pressure; FBS, fasting blood sugar; HbAlc, hemoglobin Alc; HR, heart rate; LDL-CHO, low-density lipoprotein cholesterol; T-CHO, total cholesterol.

Data are presented as mean \pm s.d.

normal range. The interval between the ultrasound and oscillometric measurement was $4.9 \pm 3.7$ days.

\section{Association between ultrasound measurements and oscillometric measurements}

Ultrasound showed upper-arm brachial artery diameter $8.5 \mathrm{~cm}$ above the antecubital fossa as $3.93 \pm 0.49 \mathrm{~mm}$. Vascular cross-sectional area measured by oscillometric measurement was $12.3 \pm 3.0 \mathrm{~mm}^{2}$. This value was converted to diameter $(3.97 \pm 0.51 \mathrm{~mm})$ for comparison with ultrasound measurements. There was no difference in brachial vascular diameter between ultrasound and oscillometric measurement $(P=0.65)$. Brachial vascular diameter derived from oscillometric measurements significantly correlated with ultrasound brachial artery diameter measurements $(r=0.75, P<0.001$, Figure 3$)$.

\section{Rest reproducibility of eA and $V_{\mathrm{E}}$ measurements}

All 16 subjects had two oscillometric measurements. The mean interval between the two oscillometric measurements was $7.2 \pm 5.2$ days. There was no significant difference in subjects' baseline condition between the first and second studies including height $(P=0.33)$, weight $(P=0.32)$, systolic BP $(P=0.64)$, diastolic BP $(P=0.35)$ or heart rate $(P=0.67)$.

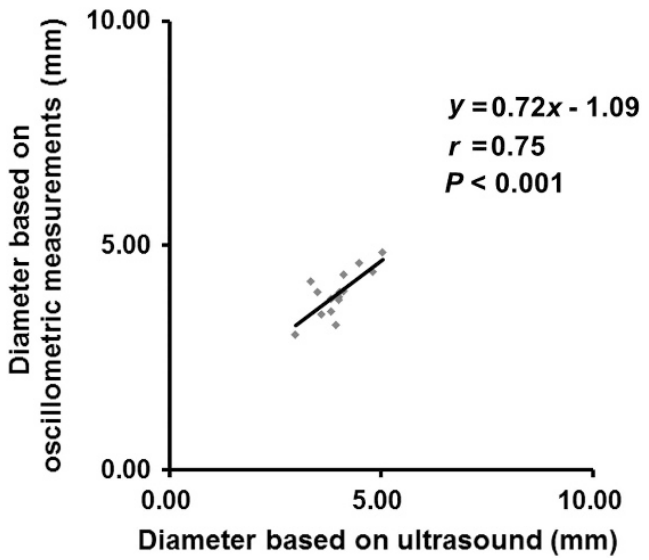

Figure 3 Correlation between ultrasound measurements and oscillometric measurements of rest brachial artery diameter. Rest estimated area $(e A)$ was converted to vascular diameter.

There was no significant difference in rest eA $(11.9 \pm 3.0$ vs. $\left.11.5 \pm 2.9 \mathrm{~mm}^{2}, P=0.32\right)$ or $V_{\mathrm{E}}(0.82 \pm 0.16$ vs. $0.77 \pm 0.16 \mathrm{~mm} \mathrm{Hg} / \%$, $P=0.09)$ between the first and second studies. Rest eA and rest $V_{\mathrm{E}}$ showed good reliability $\left(\mathrm{eA}: \mathrm{ICC}=0.88, V_{\mathrm{E}}\right.$ : ICC $\left.=0.78\right)$.

\section{NTG stress responses}

NTG administered sublingually significantly decreased systolic BP $(115.2 \pm 15.1$ to $108.2 \pm 13.9 \mathrm{~mm} \mathrm{Hg}, P=0.0004)$ and increased heart rate $(62.2 \pm 9.8$ to $67.8 \pm 7.2$ b.p.m., $P=0.002)$ in oscillometric measurements.

Ultrasound showed increasing arterial diameter after NTG administration $(4.46 \pm 0.72$ to $4.73 \pm 0.75 \mathrm{~mm}, P<0.001)$. The percentage change of arterial diameter with NTG was $15.7 \pm 5.0 \%$. eA was significantly increased after NTG administration $(12.3 \pm 3.0$ vs. $\left.17.1 \pm 4.6 \mathrm{~mm}^{2}, P<0.001\right) . V_{\mathrm{E}}$ was significantly decreased after NTG administration $(0.81 \pm 0.16$ vs. $0.65 \pm 0.11 \mathrm{~mm} \mathrm{Hg} / \%, P<0.001)$. Percentage change of eA $(\% \mathrm{eA})$ was $39.6 \pm 18.2 \%$ and percentage change of $V_{\mathrm{E}}\left(\% V_{\mathrm{E}}\right)$ was $-19.5 \pm 10.4 \%$.

However, there was no significant correlation between the percentage change of brachial artery diameter by ultrasound and the percentage eA or percentage $V_{\mathrm{E}}\left(\% \mathrm{eA}: r=0.31, P=0.25, \% V_{\mathrm{E}}\right.$ : $r=0.19, P=0.48$ ).

\section{Age-related vascular function: baseline characteristics}

In this additional analysis, we excluded two smokers because smoking attenuated vascular function. Thus, we analyzed a total of 14 subjects in this analysis.

There were 8 younger subjects ( $\leqslant 35$ years old) ${ }^{16}$ (younger group) and 6 older subjects (older group). The baseline characteristics of the two groups are addressed in Table $1 \mathrm{~b}$. As expected, the older group was older than the younger group $(P<0.001)$. Otherwise, there were no significant differences between the two groups in terms of baseline characteristics.

\section{Association between eA, $V_{\mathrm{E}}$ and aging}

There was no significant difference in the rest ultrasound measurements of brachial artery diameter between the two groups (younger: $3.79 \pm 0.38$ vs. older: $4.05 \pm 0.62, P=0.35)$. Oscillometric measurement also did not show significant differences in rest eA between the two groups (younger: $10.9 \pm 2.0 \mathrm{vs}$. older: $13.3 \pm 3.8 \mathrm{~mm}^{2}, P=0.15$ ). In contrast, the older group showed significantly higher $V_{\mathrm{E}}$ compared 
a

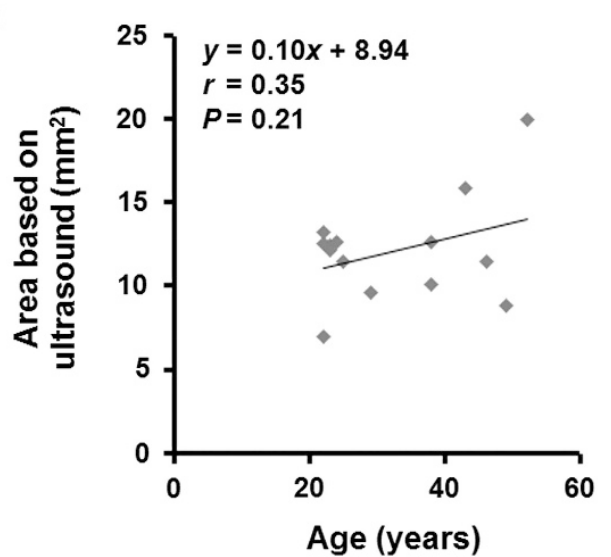

C

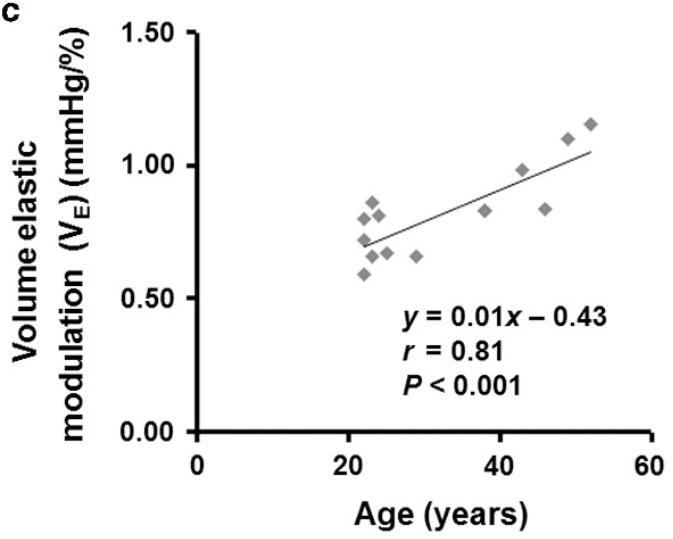

b

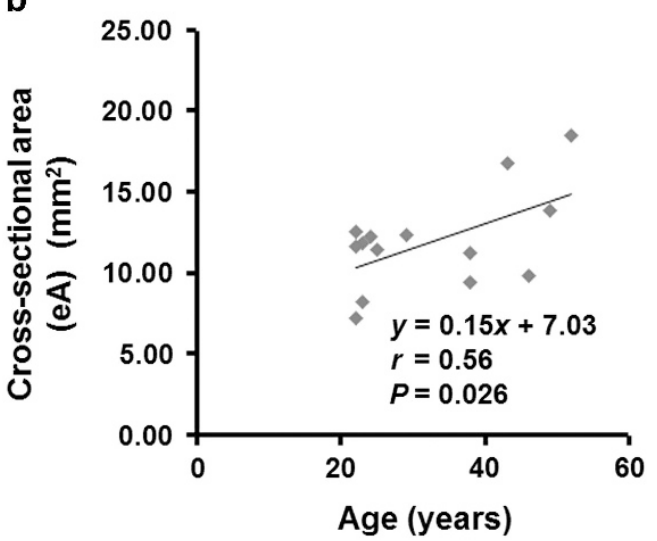

Figure 4 Correlation between age and other parameters. (a) Correlation between age and ultrasound measurements of brachial artery diameter. (b) Correlation between age and oscillometric measurement of estimated cross-sectional area (eA). (c) Correlation between age and oscillometric measurement of volume elastic modulus $\left(V_{E}\right)$.

with the younger group (younger: $0.72 \pm 0.09$ vs. older: $0.95 \pm 0.14$ $P=0.003)$.

There was no significant correlation between ultrasound-measured brachial artery diameter and age $(r=0.32, P=0.26$, Figure $4 a)$. In contrast, there was a significant correlation between eA and increasing age $(r=0.56, P=0.026$, Figure $4 \mathrm{~b})$. Rest $V_{\mathrm{E}}$ also showed significant correlation with increasing age $(r=0.81, P<0.001$, Figure $4 c)$.

\section{DISCUSSION}

The absolute value of rest brachial artery estimated cross-sectional area using oscillometric measurement showed good correlation with brachial artery ultrasound measurements. The estimated crosssectional area and $V_{\mathrm{E}}$ measurements using this new modality showed good reproducibility. Oscillometric measurement automatically detected the changes in eA and $V_{\mathrm{E}}$ after NTG administration. In addition, eA and $V_{\mathrm{E}}$ increased in association with increasing age.

Reliability of automated oscillometric measurement: comparison between oscillometric measurement and ultrasound measurements In the current system, the oscillometric method quantitatively estimates cross-sectional vascular area. In this study, we converted cross-sectional area to brachial vascular diameter in order to compare it with ultrasound measurements. The converted estimated arterial diameter was similar to that determined using ultrasound measurements. The rest estimated vascular diameter also significantly correlated with the ultrasound-measured brachial artery diameter.
Therefore, this oscillometric method should be a reliable approach and may provide similar useful anatomical information to that determined by ultrasound measurement.

Ultrasound measurement provides a visualization of the vascular lumen and therefore allows for an exact measurement of the vascular diameter, whereas oscillometric methods do not provide for such visualization and therefore provide an approximation of the vascular cross-sectional area. Given the characteristics of the oscillometric approach, soft tissues surrounding arteries may be included in crosssectional area measurements, possibly leading to an overestimation of volume. Even when this concern was taken into account, the current data showed good correlation with ultrasound measurements that are part of the standard approach. ${ }^{11}$ In fact, the current data showed the estimated brachial vascular diameter to be slightly larger than brachial artery diameter determined using ultrasound. However, there was no significant difference between the two measurements.

The cross-sectional area estimation showed high ICC. Previous data $^{23}$ reported that reproducibility of ultrasound brachial artery diameter measurement had an ICC of $0.84 .{ }^{24}$ Oscillometric measurements agree with those of previous studies. Based on these data, oscillometric measurements would be reliable and can be used in clinical or epidemiological settings.

NTG response is an endothelium-independent vascular dilatation function. ${ }^{25}$ In this study, eA significantly increased after NTG administration. The response to NTG was similar to that found using ultrasound. On the other hand, there was no correlation between the 
percentage change in diameter as measured by ultrasound and eA. The range of data for \%NTG was narrow because of the normal study population. This may be a reason for the lack of correlation in NTG response between the two tests. Oscillometric measurements may have included brachial artery and surrounding vascular areas, and therefore the value of eA may have appeared to be higher than it was found to be with ultrasound, especially under NTG stress. This also may have been the reason that there was no significant correlation between percentage eA change with NTG stress determined by oscillometric measurement and that determined by ultrasound measurement. Although we did not show the correlation, the oscillometric approach was able to detect vascular cross-sectional area change during NTG stress.

\section{Automated measurements of $V_{\mathrm{E}}$}

The rest $V_{\mathrm{E}}$ showed good reproducibility. This reproducibility is similar to that for other vascular functional evaluations. ${ }^{12}$

Arterial elastic mechanics including $V_{\mathrm{E}}$ are linked to arterial stiffness. ${ }^{7,8}$ Bank et al. ${ }^{10}$ reported that brachial artery elastic mechanics accurately evaluated vascular stiffness over a wide range of BP and vascular smooth muscle tone. Therefore, $V_{\mathrm{E}}$ should be examined in clinical practice. However, previous methods of doing so were either complex or invasive. ${ }^{10,11}$ The current oscillometric approach may build on these previous studies and may allow for practical measurements of vascular elasticity in clinical settings or epidemiological study settings.

$V_{\mathrm{E}}$ was also reduced under NTG stress. NTG dilates brachial arteries by causing smooth muscle relaxation. The oscillometric system automatically detected changing vascular tone with NTG, and these data agree with those from the previous study by Bank et al. ${ }^{10}$ The current data may imply that this approach could be used to detect anatomical and functional change during NTG stress.

\section{Relation between eA, $V_{\mathrm{E}}$ and aging}

In addition to showing the reliability of this new approach, we further looked at the association between those markers and age-related vascular anatomical and functional changes. Vascular function alters in association with aging. ${ }^{19,22}$ A previous study showed brachial artery diameter increases in relation to aging. ${ }^{1}$ In this study, the older group tended to have higher brachial vascular diameter and eA. However, the differences between the values for the younger group and those for the older group were not significant, perhaps because of the small sample size with a wider range of standard deviation. Thus, our results may partly agree with those of previous studies. Further studies are required to confirm these data in a larger study population. On the other hand, over the total study population, rest eA showed significant correlation with increasing age. These data agree with previous data acquired using the same method. ${ }^{14}$

Vascular stiffness and vascular function also alter in association with aging. ${ }^{22}$ Rahmani-Cherati et al. $^{26}$ reported that vascular elastisty was associated with vascular stiffness. Thus, attenuated $V_{\mathrm{E}}$ in the current study may indicate that older subjects had increasing vascular stiffness. The current data may agree with those of the previous study.

A previous study by Otsuka et al. ${ }^{14}$ showed very limited relationships between $V_{\mathrm{E}}$ and other cardiovascular risk factors including age, possibly due in part to the difference in the study design. In the previous study, subjects had BP measurements before oscillometric measurements and they did not have enough resting time before vascular function measurements. ${ }^{14}$ These factors may have had a significant impact on vascular function such as $V_{\mathrm{E}}{ }^{11}$ Further evaluation looking at the value of $V_{\mathrm{E}}$ measurements is required. In this study, we applied a standard approach to vascular function measurement. ${ }^{11,27}$ With the appropriate preparation, we showed that there were significant differences between the $V_{\mathrm{E}}$ measurements of the two groups. Moreover, increasing $V_{\mathrm{E}}$ was significantly correlated with increasing age. Other imaging methods such as ultrasound, positron emission tomography and pulse wave velocity showed attenuated vascular function in the older population. ${ }^{22,28,29}$ The current data agree with those from these previous studies. The current data add new insights regarding $V_{\mathrm{E}}$ to those from the previous study by Otsuka et al. ${ }^{14}$ This new method may be able to automatically detect agerelated vascular dysfunction.

The current data show an association between rest eA and $V_{\mathrm{E}}$ with increasing age. Measuring brachial artery diameter and vascular elasticity might be useful in clinical and epidemiological settings for evaluating atherosclerosis.

\section{Applied measurements model}

In the present study, we measured $V_{\mathrm{E}}$ and eA while the vessel remained circular. We applied models for thin-walled tubes. ${ }^{13}$ The current study population comprised healthy individuals whose arterial walls should be thin. Therefore, the current approach using thinwalled tube law should be appropriate.

When the vessel is compressed and buckles, measuring vascular area and diameter may become challenging. Therefore, we applied the measurement point before changing the vascular shape. However, the vessel may be buckled during measurement and hence it would be important to develop a new algorithm to estimate the cross-sectional area of the buckled vessel. This should be the next step in the development of this oscillometric measurement.

Some of the targeted study population who require vascular function measurements may have thick arterial walls. Therefore, it would be useful to add corrections factors to the thin-shelled theories ${ }^{30}$ or to apply one-dimensional general tube law as proposed by Kozlovsky et al. ${ }^{31}$

\section{Limitations}

Our study had a small population. The current protocol included two oscillometric measurements and one ultrasound study, each on a separate day (Figure 1). As each study had rest and NTG stress, it would be difficult to apply this comprehensive study protocol to a large number of subjects. Even with a small sample size, with careful preparation we showed good reproducibility of eA and $V_{\mathrm{E}}$. Although a small sample size may have had a minimal impact on the current data, we definitely need further study using a larger study population and a simplified protocol.

All study subjects were male. Women have vascular functional changes during their menstrual cycle that may have impacts on vascular function. For this initial study, we wanted to avoid these effects. However, we need to apply this measurement to women. Such studies are currently under way.

In the present study, we evaluated only normal individuals and smokers. Applying the current comprehensive protocol to coronary artery disease patients would have been difficult. However, this measurement technique should be applied to subjects with atherosclerotic risk factors and coronary artery disease patients as a next step.

In the present study, we applied the same pulse pressure, 40 $\mathrm{mm} \mathrm{Hg}$, to estimate $V_{\mathrm{E}}$. However, using individual pulse pressure would be ideal for setting P1. This possibility should be tested in the future and should be applied to this oscillometric measurement. 


\section{CONCLUSION}

This new quantitative automated oscillometric measurement accurately assessed brachial artery cross-sectional area and vascular elasticity. This measurement technique can also detect morphological and functional change under NTG stress and is a reliable approach. Therefore, this modality may have practical application in quantitatively assessing muscular artery elasticity and diameter responses.

\section{CONFLICT OF INTEREST}

The authors declare no conflict of interest.

\section{ACKNOWLEDGEMENTS}

This study was supported in part by grants from the Japanese Ministry of Education, Culture, Sports, Science and Technology (HOUGA Grant No. 24659550), Adult Vascular Disease Research Foundation (\#H22-23), Smoking Research Foundation 2011-2014 and A\&D Company (Tokyo, Japan). YT was supported by the National Institute of Radiological Sciences Human Resources Development Program (Chiba, Japan) and is supported by the Research Assistant Program, Hokkaido University Graduate School of Medicine. KY is supported by the Imura Clinical Research Award (Adult Vascular Disease Research Foundation). All data were recorded and analyzed by Hokkaido University Graduate School of Medicine.

1 Green DJ, Swart A, Exterkate A, Naylor LH, Black MA, Cable NT, Thijssen DH. Impact of age, sex and exercise on brachial and popliteal artery remodelling in humans. Atherosclerosis 2010; 210: 525-530.

2 Glagov S, Weisenberg E, Zarins CK, Stankunavicius R, Kolettis GJ. Compensatory enlargement of human atherosclerotic coronary arteries. N Engl J Med 1987; 316 1371-1375.

3 Holewijn S, den Heijer M, Swinkels DW, Stalenhoef AF, de Graaf J. Brachial artery diameter is related to cardiovascular risk factors and intima-media thickness. Eur J Clin Invest 2009; 39: 554-560.

4 Hamburg NM, Keyes MJ, Larson MG, Vasan RS, Schnabel R, Pryde MM, Mitchell GF, Sheffy J, Vita JA, Benjamin EJ. Cross-sectional relations of digital vascular function to cardiovascular risk factors in the Framingham Heart Study. Circulation 2008; 117: 2467-2474.

5 Yoshinaga K, Manabe 0, Tamaki N. Assessment of coronary endothelial function using PET. J Nucl Cardiol 2011; 18: 486-500.

6 Wohlfahrt P, Krajcoviechova A, Seidlerova J, Galovcova M, Bruthans J, Filipovsky J, Laurent S, Cifkova R. Lower-extremity arterial stiffness vs. aortic stiffness in the general population. Hypertens Res 2013; 36: 718-724.

7 Kaiser DR, Mullen K, Bank AJ. Brachial artery elastic mechanics in patients with heart failure. Hypertension 2001; 38: 1440-1445.

8 O'Rourke MF, Staessen JA, Vlachopoulos C, Duprez D, Plante GE. Clinical applications of arterial stiffness; definitions and reference values. Am J Hypertens 2002; 15 426-444.

9 Clairotte C, Retout S, Potier L, Roussel R, Escoubet B. Automated ankle-brachial pressure index measurement by clinical staff for peripheral arterial disease diagnosis in nondiabetic and diabetic patients. Diabetes Care 2009; 32: 1231-1236.

10 Bank AJ, Wang H, Holte JE, Mullen K, Shammas R, Kubo SH. Contribution of collagen, elastin, and smooth muscle to in vivo human brachial artery wall stress and elastic modulus. Circulation 1996; 94: 3263-3270.

11 Corretti MC, Anderson TJ, Benjamin EJ, Celermajer D, Charbonneau F, Creager MA Deanfield J, Drexler H, Gerhard-Herman M, Herrington D, Vallance P, Vita J, Vogel RInternational Brachial Artery Reactivity Task F. Guidelines for the ultrasound assessment of endothelial-dependent flow-mediated vasodilation of the brachial artery: a report of the International Brachial Artery Reactivity Task Force. J Am Coll Cardiol 2002; 39: 257-265

12 Kinlay S, Creager MA, Fukumoto M, Hikita H, Fang JC, Selwyn AP, Ganz P. Endothelium-derived nitric oxide regulates arterial elasticity in human arteries in vivo. Hypertension 2001; 38: 1049-1053.

13 Whittaker RJ, Heil M, Jensen $\mathrm{OE}$, Waters SL. A rational derivation of a tube law from shell theory. Q J Mech Appl Math 2010; 63: 465-496.
14 Otsuka T, Munakata R, Kato K, Kodani E, Ibuki C, Kusama Y, Seino Y, Kawada T. Oscillometric measurement of brachial artery cross-sectional area and its relationship with cardiovascular risk factors and arterial stiffness in a middle-aged male population. Hypertens Res 2013; 36: 910-915.

15 Diamond GA, Forrester JS. Analysis of probability as an aid in the clinical diagnosis of coronary-artery disease. N Engl J Med 1979; 300: 1350-1358.

16 Zimmerman FH, Cameron A, Fisher LD, Ng G. Myocardial infarction in young adults: angiographic characterization, risk factors and prognosis (Coronary Artery Surgery Study Registry). J Am Coll Cardiol 1995; 26: 654-661.

17 Fukui M, Ushigome E, Tanaka M, Hamaguchi M, Tanaka T, Atsuta H, Ohnishi M, Oda Y, Hasegawa G, Nakamura N. Could home arterial stiffness index be a novel marker for arterial stiffness in patients with type 2 diabetes? Hypertens Res 2013; 36 645-649.

18 Hu H, Cui H, Han W, Ye L, Qiu W, Yang H, Zhang C, Guo X, Mao G. A cutoff point for arterial stiffness using the cardio-ankle vascular index based on carotid arteriosclerosis. Hypertens Res 2013; 36: 334-341.

19 Doonan RJ, Mutter A, Egiziano G, Gomez YH, Daskalopoulou SS. Differences in arterial stiffness at rest and after acute exercise between young men and women. Hypertens Res 2013; 36: 226-231.

20 Hamburg NM, Palmisano J, Larson MG, Sullivan LM, Lehman BT, Vasan RS, Levy D, Mitchell GF, Vita JA, Benjamin EJ. Relation of brachial and digital measures of vascular function in the community: the Framingham heart study. Hypertension 2011; 57 390-396.

21 Liu L, Ruddy TD, Dalipaj M, Szyszkowicz M, You H, Poon R, Wheeler A, Dales R. Influence of personal exposure to particulate air pollution on cardiovascular physiology and biomarkers of inflammation and oxidative stress in subjects with diabetes. J Occup Environ Med 2007; 49: 258-265.

22 Celermajer DS, Sorensen KE, Spiegelhalter DJ, Georgakopoulos D, Robinson J, Deanfield JE. Aging is associated with endothelial dysfunction in healthy men years before the age-related decline in women. J Am Coll Cardiol 1994; 24 471-476.

23 Cohen J. A power primar. Psychol Bull 1992; 112: 155-159.

24 Meirelles Cde M, Leite SP, Montenegro CA, Gomes PS. Reliability of brachial artery flow-mediated dilatation measurement using ultrasound. Arq Bras Cardiol 2007; 89: 160-167 176-183.

25 Celermajer DS, Adams MR, Clarkson P, Robinson J, McCredie R, Donald A, Deanfield JE. Passive smoking and impaired endothelium-dependent arterial dilatation in healthy young adults. N Engl J Med 1996; 334: 150-154.

26 Rahmani-Cherati T, Mokhtari-Dizaji M, Gity M. Association between atherosclerosis in carotid artery and elastic modulus of brachial artery. J Teh Univ Heart Ctr 2006; 1 15-19.

27 Weber MA, Schiffrin EL, White WB, Mann S, Lindholm LH, Kenerson JG, Flack JM, Carter BL, Materson BJ, Ram CV, Cohen DL, Cadet JC, Jean-Charles RR, Taler S, Kountz D, Townsend R, Chalmers J, Ramirez AJ, Bakris GL, Wang J, Schutte AE, Bisognano JD, Touyz RM, Sica D, Harrap SB. Clinical practice guidelines for the management of hypertension in the community a statement by the American Society of Hypertension and the International Society of Hypertension. J Hypertens 2014; 32 3-15.

28 Uren NG, Camici PG, Melin JA, Bol A, de Bruyne B, Radvan J, Olivotto I, Rosen SD, Impallomeni M, Wijns W. Effect of aging on myocardial perfusion reserve. J Nucl Med 1995; 36: 2032-2036.

29 Alghatrif M, Strait JB, Morrell CH, Canepa M, Wright J, Elango P, Scuteri A, Najiar SS, Ferrucci L, Lakatta EG. Longitudinal trajectories of arterial stiffness and the role of blood pressure: the Baltimore Longitudinal Study of Aging. Hypertension 2013; 62 934-941.

30 Marzo A, Luo XY, Bertram CD. Three-dimensional collapse and steady flow in thickwalled flexible tubes. J Fluid Struct 2005; 20: 817-835.

31 Kozlovsky P, Zaretsky U, Jaffa AJ, Elad D. General tube law for collapsible thin and thick-wall tubes. J Biomech 2014; 47: 2378-2384.

(1) (\$) $\Theta$ This work is licensed under a Creative Commons Attribution-NonCommercial-NoDerivs $\quad 4.0$ International License. The images or other third party material in this article are included in the article's Creative Commons license, unless indicated otherwise in the credit line; if the material is not included under the Creative Commons license, users will need to obtain permission from the license holder to reproduce the material. To view a copy of this license, visit http://creativecommons.org/licenses/ by-nc-nd/4.0/ 